\title{
Bifurcation analysis of nephron pressure and flow regulation
}

\author{
Barfred, Mikael; Mosekilde, Erik; Holstein-Rathlou, N.-H.
}

\section{Published in:}

Chaos

Link to article, DOI:

10.1063/1.166175

Publication date:

1996

Document Version

Publisher's PDF, also known as Version of record

Link back to DTU Orbit

Citation (APA):

Barfred, M., Mosekilde, E., \& Holstein-Rathlou, N-H. (1996). Bifurcation analysis of nephron pressure and flow regulation. Chaos, 6(3), 280-287. https://doi.org/10.1063/1.166175

\section{General rights}

Copyright and moral rights for the publications made accessible in the public portal are retained by the authors and/or other copyright owners and it is a condition of accessing publications that users recognise and abide by the legal requirements associated with these rights.

- Users may download and print one copy of any publication from the public portal for the purpose of private study or research.

- You may not further distribute the material or use it for any profit-making activity or commercial gain

- You may freely distribute the URL identifying the publication in the public portal

If you believe that this document breaches copyright please contact us providing details, and we will remove access to the work immediately and investigate your claim. 


\title{
Bifurcation analysis of nephron pressure and flow regulation
}

\author{
Mikael Barfred and Erik Mosekilde \\ Center for Chaos and Turbulence Studies, Department of Physics, The Technical University of Denmark, \\ 2800 Lyngby, Denmark \\ Niels-Henrik Holstein-Rathlou \\ Department of Medical Physiology, Panum Institute, The University of Copenhagen, 2200 Copenhagen N, \\ Denmark
}

(Received 4 March 1996; accepted for publication 25 July 1996)

\begin{abstract}
One- and two-dimensional continuation techniques are applied to study the bifurcation structure of a model of renal flow and pressure control. Integrating the main physiological mechanisms by which the individual nephron regulates the incoming blood flow, the model describes the interaction between the tubuloglomerular feedback and the response of the afferent arteriole. It is shown how a Hopf bifurcation leads the system to perform self-sustained oscillations if the feedback gain becomes sufficiently strong, and how a further increase of this parameter produces a folded structure of overlapping period-doubling cascades. Similar phenomena arise in response to increasing blood pressure. The numerical analyses are supported by existing experimental results on anesthetized rats. (C) 1996 American Institute of Physics. [S1054-1500(96)02503-7]
\end{abstract}

\begin{abstract}
The function of the kidneys plays an essential role for the regulation of the blood pressure and hence for the development of cardiovascular diseases. At the same time, the kidneys dispose of a variety of mechanisms to protect their own function against variations in the blood pressure. Experiments on rats have shown that these mechanisms can lead to self-sustained oscillations, period doublings and chaos. Based on a detailed physiological knowledge we have developed a model that can reproduce these findings. A two-parameter bifurcation analysis of the model shows a so-called crossroad structure of overlying period-doubling bifurcations previously observed for one- and two-dimensional mappings.
\end{abstract}

\section{INTRODUCTION}

Physiological control systems represent an interesting area of application for the ideas and techniques of nonlinear science. Investigations performed during the last decades have revealed the existence of a great variety of biological rhythms with periods ranging from fractions of a second to hours or even weeks, ${ }^{1}$ and oscillatory and pulsatile phenomena are increasingly recognized as essential for the control and function of normal physiological systems. ${ }^{2}$ Examples are hormonal regulation, ${ }^{3-5}$ and cell-to-cell signaling, ${ }^{6}$ with the secretion of pituitary and other hormones typically occurring in pulses with $1-3 \mathrm{~h}$ intervals.

The present analysis is concerned with nonlinear phenomena arising through interaction of the main control processes in the nephron, the functional unit of the kidney. By regulating the excretion of salts, water, and metabolic end products, the kidneys play an important role in maintaining a suitable environment for the cells of the body. In particular, the kidneys control the composition and volume of the extracellular fluid and the blood pressure.

To protect their own function and secure a relatively constant blood flow, the kidneys also dispose of mechanisms that can compensate for variations in arterial blood pressure. It has long been recognized that this ability partly rests with controls in the individual nephron, primarily the so-called tubuloglomerular feedback (TGF). ${ }^{7}$ This is a negative feedback that regulates the diameter of the afferent arteriole, and hence the incoming blood flow, in dependence of the chloride concentration of the tubular fluid leaving the loop of Henle.

Early experiments by Leyssac and Baumbach ${ }^{8}$ and by Leyssac and Holstein-Rathlou ${ }^{9,10}$ demonstrated that the feedback regulation could become unstable and generate selfsustained oscillations in the proximal intratubular pressure with characteristic periods of 30-40 s. While for normal rats the oscillations had the typical appearance of a limit cycle, highly irregular oscillations were found for spontaneously hypertensive rats (SHR). Oscillations were observed for $50 \%-80 \%$ of the investigated nephrons. For the individual nephron they could last for half an hour or more. For nonoscillatory nephrons, self-sustained oscillations could be elicited by microperfusion with artificial tubular fluid, i.e., by artificially increasing the rate of flow through the loop of Henle. Already in these early studies, the irregular oscillatory patterns observed for SHR were suggested to be the result of a chaotic process. It has subsequently been observed that similar irregular oscillations can develop for normal rats, if the arterial pressure is increased by reducing the blood supply to the other kidney. ${ }^{11}$

By integrating existing but hitherto separate physiological descriptions of glomerular filtration, tubular dynamics, and tubuloglomerular feedback, initial modeling studies by Holstein-Rathlou and Leyssac ${ }^{12}$ and by Jensen et al. ${ }^{13}$ were able to show that the total feedback system for physiologically realistic parameter values can become unstable and exhibit self-sustained oscillations with the experimentally observed period. When introducing a phenomenologically motivated nonlinearity in the response of the afferent 
arteriole, ${ }^{13}$ the model produced a transition to chaos through a cascade of period-doubling bifurcations as either the delay in the feedback or the steepness of the control characteristics were increased.

A subsequent study by Jensen et al. ${ }^{14}$ included experimental results on period doubling in the tubular pressure oscillations. This study also provided results for the fractal dimension of the pressure variations both for normo- and hypertensive rats. Finally, in the study a model for the interaction of neighboring nephrons was discussed. Experimental results on synchronization of nearby nephrons were obtained by Holstein-Rathlou. ${ }^{15} \mathrm{He}$ also observed how microperfusion of one nephron affected the amplitude of the pressure oscillations in a neighboring nephron, clearly demonstrating the existence of a coupling between the functional units.

In 1990, Holstein-Rathlou and Marsh ${ }^{16,17}$ made a number of new contributions toward a detailed understanding of the oscillatory TGF mechanism. On the experimental side they succeeded in simultaneously recording data for the proximal and distal tubular pressure and for the chloride concentration of the liquid leaving the loop of Henle. This provided a clear picture of the relative amplitudes of the various signals as well as of their phase relations. On the theoretical side they introduced a set of coupled partial differential equations to describe the propagation of the pressure oscillations and the associated exchanges of water and salts along the tubule. Compared to the model suggested by Jensen et al. ${ }^{13}$ the description of the response of the afferent arteriole was simplified, and the model no longer produced period doublings and chaos.

With this exception, the spatially extended model seemed capable of explaining the experimental findings related to the self-sustained tubular oscillations. To test if the tubuloglomerular feedback could fully account for renal autoregulation at the level of individual nephrons, a series of experiments were performed in which broadband noise was applied to the arterial pressure. ${ }^{18}$ When comparing the noise response of the model to the response of the rat nephron, it became clear, however, that while the model correctly predicted the phase and amplitude characteristics at frequencies below approximately $70 \mathrm{mHz}$, the experiments exhibited a significant peak in the noise response at higher frequencies that was no accounted for in the model. Here, an additional control mechanism had to be in operation. Holstein-Rathlou and Marsh proposed ${ }^{19}$ that this high-frequency regulation was associated with the myogenic response of the afferent arteriole.

Based on in vitro measurements of the stress-strain relationship for muscle strips, Feldberg ${ }^{20}$ recently developed a detailed description of the myogenic regulation and of its interaction with the TGF mechanism. A characteristic feature of this model is that the afferent arteriole tends to contract in response to increasing hydrostatic pressure in the blood. This gives rise to a new potential instability that operates in the $100 \mathrm{mHz}$ regime.

The purpose of the present study is, by means of oneand two-dimensional continuation techniques, to examine the bifurcation structure of a model that combines the TGF feed-

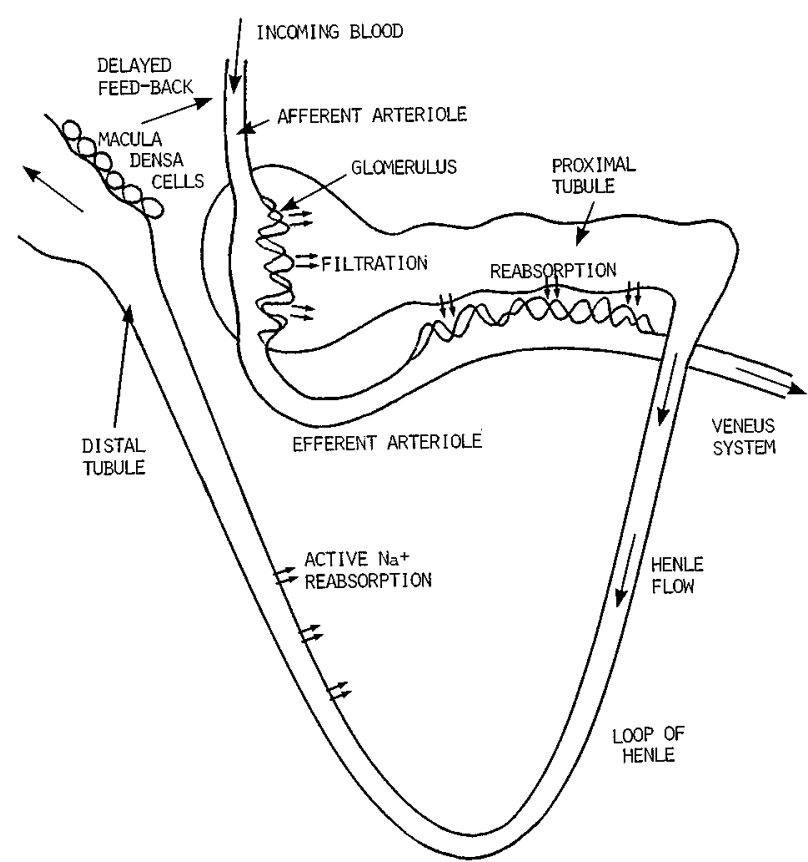

FIG. 1. A schematic drawing of a nephron with its glomerulus, proximal tubule, loop of Henle, and distal tubule. The diameter of the tubule is typically $20 \mu \mathrm{m}$. Measurements of the proximal and distal tubular pressure and of the distal tubular chloride concentration are performed on anesthetized rats.

back mechanism with a detailed account of the response of the afferent arteriole. Our investigations reveal the existence of several overlying sheets of period-doubling cascades. Hence, when combining the TGF and the nonlinear arteriolar responses, the model again allows for the occurrence of chaos as the arterial pressure or the feedback in the TGF mechanism is increased. Now, however, the results are obtained on a much more satisfactory physiological basis.

\section{NEPHRON PRESSURE AND FLOW REGULATION}

Figure 1 illustrates the main structure of the nephron. Blood enters the system through the afferent arteriole, a short vessel that is capable of regulating the blood flow by varying its diameter. At the glomerulus the blood passes a system of $20-40$ parallel capillary loops, where $25 \%-35 \%$ of the water together with blood constituents with molecular weight below 68000 is filtered out into the proximal tubule. Blood cells and proteins are retained, and the filtration process saturates when the colloid osmotic pressure balances the hydrostatic pressure difference between the blood and the filtrate in the tubule. The blood leaves the glomerulus through the efferent arteriole to pass through a new capillary bed and out into the venous system. In the second capillary bed, which embraces the tubule, a nearly constant fraction of the filtrate is reabsorbed.

The nephron itself is a hollow tube with a membrane wall formed by a single layer of highly specialized epithelial cells. With its various sections: the proximal tubule, the loop of Henle, the distal tubule, and the collecting duct, the total length of the nephron is approximately $2 \mathrm{~cm}$. The inner di- 
ameter, however, is only about $20 \mu \mathrm{m}$. The proximal tubule is located within the outer layer of the kidney, the cortex, and is hence accessible for pressure measurements by means of glass micropipettes. From the proximal tubule the nephron traverses down through the renal medulla, forming the descending and ascending limb of the loop of Henle. When reentering the cortex, the ascending limb changes tissue type to become the distal tubule. The interesting anatomical feature that the terminal part of the ascending limb passes immediately by the afferent arteriole of the same nephron forms the basis for the tubuloglomerular feedback. At the point of contact, specialized tubular cells (macula densa cells) monitor the composition of the tubular fluid and produce a signal that acts on the smooth muscle cells in the wall of the afferent arteriole.

Except that there are no large molecules, the composition of the ultrafiltrate in the glomerulus is like that of the blood plasma. Without changing the composition much, the proximal tubule reabsorbs approximately two-thirds of the water and salts. As the filtrate flows into the descending limb of the loop of Henle, the concentration of $\mathrm{NaCl}$ in the interstitial fluid surrounding the tubule increases significantly, and osmotic processes cause a reabsorption of water. At the same time salts and metabolic end products are secreted into the tubular fluid. The ascending limb, on the other hand, is practically impermeable to water, and its epithelial cells contain molecular pumps that transport sodium and chloride from the tubular fluid into the surrounding interstitium. In this way the $\mathrm{NaCl}$ concentration of the tubular fluid is again reduced.

Near the terminal part of the loop of Henle, the macula densa cells monitor the $\mathrm{NaCl}$ concentration of the tubular fluid and produce a feedback signal to the afferent arteriole. When the Henle flow is high, the $\mathrm{Na}^{+}$and $\mathrm{Cl}^{-}$concentrations at the macula densa will also be high, and the feedback causes the afferent arteriole to contract. This reduces the glomerular filtration pressure and hence the Henle flow. Due to a finite transition time through the tubular system, the salt concentration at the macula densa does not change instantaneously upon a change in the Henle flow. An additional lag is associated with transmission of the signal from the macula densa cells to the smooth muscle cells in the arteriolar wall, presumably because this transmission involves a cascade of processes. A characteristic value of this delay for a normal rat is $4-5 \mathrm{~s}^{21}$

The steady-state response of the arteriolar resistance to changes in the Henle flow has been worked out from openloop experiments. ${ }^{22}$ In a typical setup a paraffin block is inserted into the middle of the proximal tubule with micropipettes on either side, and the magnitude of the filtration rate is measured as a function of the externally forced flow of artificial tubular fluid into the loop of Henle. The response typically follows an $\mathrm{S}$-shaped characteristic ${ }^{23}$ expressing the physiological restrictions on both the maximal and the minimal arteriolar diameter. Like the delay in the tubuloglomerular feedback, the steepness of this characteristic plays a major role for the stability of the feedback regulation. This parameter varies significantly between normal and hyperten- sive rats, with the hypertensive rats displaying the steeper slopes.

Besides reacting to the TGF signal, the afferent arteriole also responds to changes in its transmural pressure. A similar, so-called myogenic, response appears to be involved in the autoregulation of the blood flow to many other organs. To protect the capillaries and small vessels against fluctuations in blood pressure, and to secure a relatively constant blood supply, the myogenic response causes the arterioles to contract when the arterial pressure increases. Despite much research, the detailed mechanisms of this response are still unknown. Based on in vitro experiments on the strain-stress relationship for muscle strips, Feldberg et al. ${ }^{20,24}$ have proposed a mathematical model of the reaction of the arteriolar wall. This reaction is considered to consist of a passive elastic component in parallel with an active muscular component.

In the present paper we have used a formulation to develop a more satisfactory description of the nonlinear response of the arteriolar wall to the feedback signal from the macula densa cells. One- and two-dimensional continuation techniques have then been applied to study the bifurcation structure of the complete model. We show how a Hopf bifurcation leads the system to perform self-sustained oscillations when the feedback gain becomes sufficiently strong. A further increase of this parameter is found to produce a folded structure of overlapping period-doubling cascades much like the swallow tail or shrimp structures observed in the Chua circuit ${ }^{25}$ and in bimodal one-dimensional maps. ${ }^{26,27}$ Detailed mathematical analyses of such structures have been performed, for instance, by Carcasses et al. ${ }^{28}$ by Kuznetsov et al. ${ }^{29}$ and by MacKay and Tresser. ${ }^{30}$

\section{MODEL OF NEPHRON AUTOREGULATION}

In our dynamic model, the proximal tubule is considered an elastic structure with little or no flow resistance. The tubular pressure $P_{t}$ changes in response to differences between the in- and outflows,

$$
\frac{d P_{t}}{d t}=\frac{1}{C_{\text {tub }}}\left[F_{\text {filt }}-F_{\text {reab }}-F_{\text {Hen }}\right] .
$$

Here, $F_{\text {filt }}$ is the single nephron glomerular filtration rate and $C_{\text {tub }}$ the elastic compliance of the tubule. The Henle flow,

$$
F_{\text {Hen }}=\frac{P_{t}-P_{d}}{R_{\text {Hen }}},
$$

is determined by the difference between the proximal $\left(P_{t}\right)$ and the distal $\left(P_{d}\right)$ tubular pressures and by the flow resistance $R_{\mathrm{Hen}}$ in the loop of Henle. This description is clearly a simplification, since a significant reabsorption of water and salts occurs during passage of the loop of Henle. The reabsorption $F_{\text {reab }}$ in the proximal tubule and the flow resistance $R_{\text {Hen }}$ are assumed to be constant.

The glomerular filtration rate is expressed as ${ }^{12}$ 


$$
F_{\text {filt }}=\left(1-H_{a}\right)\left(1-\frac{C_{a}}{C_{e}}\right) \frac{P_{a}-P_{g}}{R_{a}},
$$

where $H_{a}$ is the afferent hematocrit (i.e., the fraction that the blood cells constitute of the total blood volume at the entrance to the glomerular capillaries). $C_{a}$ and $C_{e}$ are the concentrations of protein in the afferent and efferent plasma, respectively, $P_{a}$ and $P_{g}$ are the arterial and glomerular blood pressures, respectively, and $R_{a}$ is the hemodynamic resistance of the afferent arteriole. $\left(P_{a}-P_{g}\right) / R_{a}$ determines the incoming blood flow. Multiplied by $\left(1-H_{a}\right)$ this gives the plasma flow. Finally, the factor $\left(1-C_{a} / C_{e}\right)$ relates the filtration rate to the change in protein concentration for the plasma remaining in the vessel.

The glomerular pressure $P_{g}$ is determined by distributing the arterial to venous pressure drop between the afferent and the efferent arteriolar resistance, i.e., as the solution to the linear equation

$$
P_{g}=P_{v}+R_{e}\left(\frac{P_{a}-P_{g}}{R_{a}}-F_{\text {filt }}\right),
$$

where the venous pressure $P_{v}$ and the efferent arteriolar resistance $R_{e}$ are considered as constants.

The protein concentration $C_{e}$ in the efferent blood is determined from the assumption that filtration equilibrium is established before the blood leaves the glomerular capillaries, i.e., that the glomerular hydrostatic pressure minus the efferent colloid osmotic pressure $P_{\text {osm }}$ equals the tubular pressure. By virtue of a nonlinear relation between the osmotic pressure and the protein concentration $C$,

$$
P_{\text {osm }}=a C+b C^{2},
$$

this leads to an expression of the form

$$
C_{e}=\frac{1}{2 b}\left[\sqrt{a^{2}-4 b\left(P_{t}-P_{g}\right)}-a\right] .
$$

In the computer model the simultaneous equations (3), (4), and (6) are combined into a single third-order equation for $C_{e}$. For relevant values of the various parameters, this equation has a single, positive solution. This solution is determined numerically for each integration step.

The glomerular feedback is described by an empirically based sigmoidal relation between the activation $\psi$ of the afferent arteriole and the delayed Henle flow $\chi_{3}$,

$$
\psi=\psi_{\max }-\frac{\psi_{\max }-\psi_{\min }}{1+\exp \left[\alpha\left(3 \chi_{3} / T F_{\mathrm{Hen} 0}-S\right)\right]} .
$$

Here, $\psi_{\max }$ and $\psi_{\min }$ denote, respectively, the maximum and the minimum values of the activation. $\alpha$ determines the slope of the feedback curve, and $S$ the displacement of the curve along the flow axis. $F_{\text {Hen } 0}$ is a normalization value for the Henle flow.

The delay in the tubuloglomerular feedback, arising both from the transit time through the loop of Henle and from the cascaded processes between the macula densa cells and the smooth muscle cells in the arteriolar wall, is represented by means of three first-order coupled differential equations,

$$
\begin{aligned}
& \frac{d \chi_{1}}{d t}=F_{\mathrm{Hen}}-\frac{3}{T} \chi_{1}, \\
& \frac{d \chi_{2}}{d t}=\frac{3}{T}\left(\chi_{1}-\chi_{2}\right), \\
& \frac{d \chi_{3}}{d t}=\frac{3}{T}\left(\chi_{2}-\chi_{3}\right),
\end{aligned}
$$

with $T$ being the total delay time. This representation implies that the delay is represented as a smoothed process, with $\chi_{1}$, $\chi_{2}$, and $\chi_{3}$ being intermediate variables in the delay chain.

The afferent arteriole is divided into two serially coupled sections of which the first (representing a fraction $\beta$ of the total length) is assumed to have a constant hemodynamic resistance, while the second (closer to the glomerulus) is capable of varying its diameter and hence the flow resistance in dependence of the tubuloglomerular feedback activation, ${ }^{20}$

$$
R_{a}=R_{a 0}\left[\beta+(1-\beta) r^{-4}\right] .
$$

Here, $R_{a 0}$ denotes a normal value of the arteriolar resistance and $r$ is the radius of the active part of the vessel, normalized relatively to its resting value.

Experiments have shown that arterioles tend to perform damped, oscillatory contractions in response to external stimuli. ${ }^{17,31}$ This may be described by means of a secondorder differential equation of the form

$$
\frac{d^{2} r}{d t^{2}}+d \frac{d r}{d t}-\frac{P_{\mathrm{av}}-P_{\mathrm{eq}}}{\omega}=0 .
$$

Here, $d$ is a characteristic time constant describing the damping of the oscillations and $\omega$ is an effective mass-to-elasticity ratio for the arteriolar wall,

$$
P_{\mathrm{av}}=\frac{1}{2}\left(P_{a}-\left(P_{a}-P_{g}\right) \beta \frac{R_{a 0}}{R_{a}}+P_{g}\right)
$$

is the average pressure in the active part of the arteriole, and $P_{\text {eq }}$ is the value of this pressure for which the arteriole is in equilibrium with its present radius and the present muscular activation.

The reaction of the arterial wall to changes in the blood pressure is considered to consist of a passive, elastic component in parallel with an active, muscular response. The elastic component is determined by the properties of the connective tissue, which consists mostly of collagen and elastin. The relation between strain $\epsilon$ and elastic stress $\tau_{e}$ for homogeneous soft tissue may be described as ${ }^{32}$

$$
\tau_{e}=C_{0}\left(e^{\gamma \epsilon}-1\right),
$$

where $C_{0}$ and $\gamma$ are constants characterizing the tissue. For very small values of $\epsilon(\gamma \epsilon \ll 1)$, we have a linear strain-stress relation. However, for larger $\epsilon$ values, the stress rises exponentially with the strain.

The active stress-strain relation due to contraction of the vascular smooth muscles appears to be surprisingly simple. ${ }^{33}$ For some $\epsilon_{\max }$ the active stress $\tau_{a}$ is maximum, and on both sides the stress decreases almost linearly with $\left|\epsilon-\epsilon_{\max }\right|$. Moreover, the stress is proportional to the muscle tone $\psi$. By 


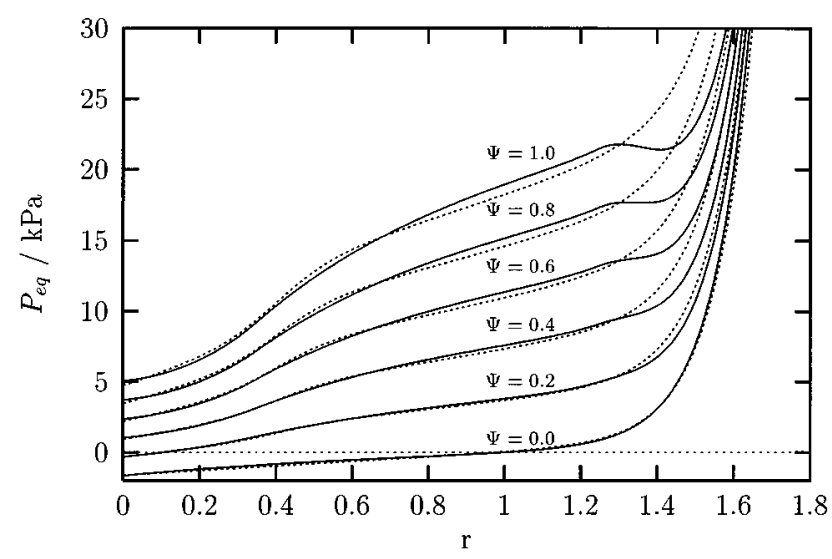

FIG. 2. Relation between the equilibrium transmural pressure $P_{\mathrm{eq}}$ and the normalized arteriolar radius $r$ for different values of the muscular activation $\psi$. The dotted curves represent the analytic approximation applied in the present study.

numerically integrating the passive and active contributions to the response across the arteriolar wall, one can establish a relation between the equilibrium pressure $P_{\text {eq }}$, the normalized radius $r$, and the activation level $\psi .^{20,24}$ This relation is represented by the fully drawn curves in Fig. 2. As an approximation to these curves, represented by dotted lines in Fig. 2, we have applied an analytic expression of the form

$$
\begin{aligned}
P_{\text {eq }}= & 2.4 \times e^{10(r-1.4)}+1.6(r-1) \\
& +\psi\left(\frac{4.7}{1+e^{13(0.4-r)}}+7.2(r+0.9)\right),
\end{aligned}
$$

where $P_{\text {eq }}$ is expressed in $\mathrm{kPa}\left(1 \mathrm{kPa} \triangleq 10^{3} \mathrm{~N} / \mathrm{m}^{2} \cong 7.5\right.$ $\mathrm{mm} \mathrm{Hg}$ ). The first two terms in (15) represent the pressure versus radius relation for the nonactivated arteriole. It consists of an exponential and a linear term arising from the two terms in the expression (14) for $\tau_{e}$. The terms proportional to $\psi$ represent the active response. This is approximately given by a sigmoidal term superimposed onto a linear term. The activation from the TGF mechanism is assumed to be given by (7).

The above equations complete the description of our model. In total we have six coupled ordinary differential equations, each representing an essential physiological relation. Because of the need to numerically evaluate $C_{e}$ in each integration step, the model cannot be brought onto an explicit form. The applied parameters are specified in Table I.

TABLE I. List of model parameters.

\begin{tabular}{lc}
\hline \hline$P_{a}=13.3 \mathrm{kPa}$ & $C_{a}=54 \mathrm{~g} / \mathrm{l}$ \\
$P_{v}=1.3 \mathrm{kPa}$ & $a=21.7 \mathrm{~Pa}(\mathrm{l} / \mathrm{g})$ \\
$P_{d}=0.6 \mathrm{kPa}$ & $b=0.39 \mathrm{~Pa}(1 / \mathrm{g})^{2}$ \\
$R_{a}=2.4 \mathrm{kPa}(\mathrm{s} / \mathrm{nl})$ & $\omega=20 \mathrm{kPa} \mathrm{s}$ \\
$R_{e}=1.9 \mathrm{kPa}(\mathrm{s} / \mathrm{nl})$ & $d=0.04 / \mathrm{s}$ \\
$R_{\mathrm{Hen}}=5.3 \mathrm{kPa}(\mathrm{s} / \mathrm{nl})$ & $\beta=0.67$ \\
$C_{\text {tub }}=3.0 \mathrm{nl} / \mathrm{kPa}$ & $\psi_{\min }=0.20$ \\
$H_{a}=0.5$ & $\psi_{\max }=0.44$ \\
$F_{\text {reab }}=0.3 \mathrm{nl} / \mathrm{s}$ & $\psi_{\mathrm{eq}}=0.38$ \\
$F_{\text {Hen } 0}=0.2 \mathrm{nl} / \mathrm{s}$ & \\
\hline \hline
\end{tabular}

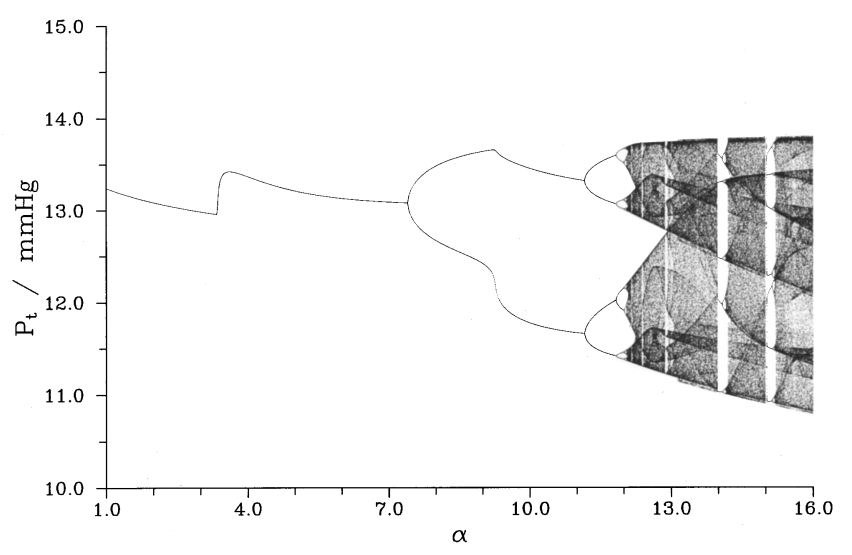

FIG. 3. Brute force bifurcation diagram for the nephron model for a delay in the tubuloglomerular feedback $T=3.0 \mathrm{~s}$. The period-doubling cascade accumulates approximately at $\alpha=12.0$. Note the abrupt displacement of the period-2 solution at $\alpha \cong 9.3$.

They have all been adopted from Jensen et al. ${ }^{13}$ where the origin of each parameter is discussed.

\section{BIFURCATION STRUCTURE}

Figure 3 shows a brute force bifurcation diagram obtained for the nephron model when scanning the steepness $\alpha$ of the response characteristics while keeping the delay in the tubuloglomerular feedback constant at $T=3.0 \mathrm{~s}$. The integration was performed with a fourth/fifth order Runge-Kutta pair with automatic time step control. For small values of $\alpha$, the model displays a stable equilibrium solution. At $\alpha \cong 3.4$, a supercritical Hopf bifurcation takes place, and for $\alpha \cong 7.5$ a period-doubling sequence is initiated, accumulating with the development of chaos around $\alpha=12.0$. Similar results can be obtained by varying the arterial pressure $P_{a}$ or the feedback delay $T$.

On a qualitative level these results agree both with the observation of period doubling in the response of the nephron to an external disturbance, ${ }^{14}$ and with the observation of chaos in normotensive rats made hypertensive by clipping one of the renal arteries. ${ }^{11}$ Genetically hypertensive rats (SHR) are supposed to have significantly larger $\alpha$ values than normal rats, and the model also agrees with the observation that these rats usually display chaotic pressure variations. $^{9}$

Figure 4 shows the phase plot of the chaotic attractor obtained for $T=3.0 \mathrm{~s}$ and $\alpha=14.5$. Here, we have displayed the normalized arteriolar radius $r$ against the proximal tubular pressure $P_{t}$. With physiologically realistic parameter values the model reproduces the observed oscillatory behavior for the tubular pressure with characteristic periods of 20-30 s. The amplitude in the pressure variations also correspond to the experimentally obtained values.

At $\alpha \cong 9.3$, the bifurcation diagram in Fig. 3 shows an abrupt displacement of the period-2 solution. Calculation of the characteristic multipliers shows that one of these comes close to 1.0 , but never reaches the unit circle. Hence, there is no bifurcation in this point, but the feature arises because the 


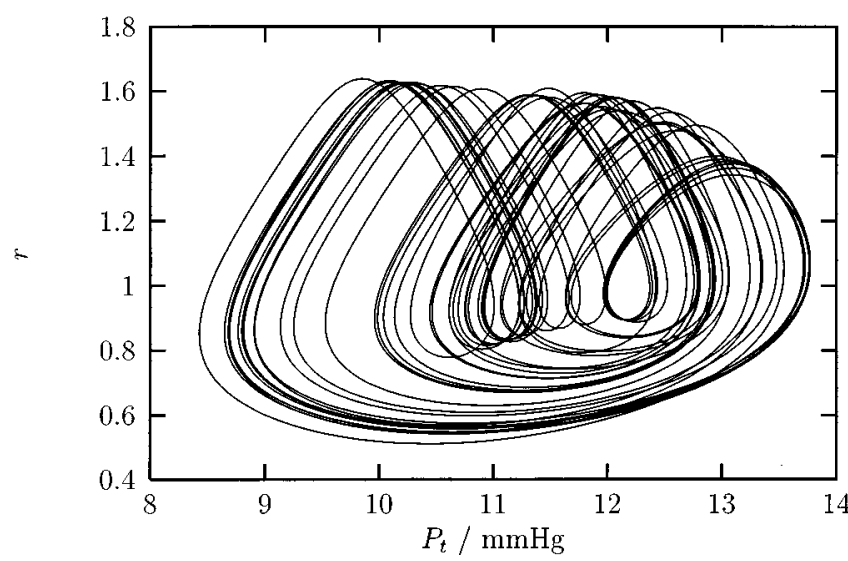

FIG. 4. Phase plot for the chaotic solution obtained for $\alpha=14.5$ and $T=3.0$ s. The normalized arteriolar radius $r$ is displayed versus the proximal tubular pressure $P_{t}$.

scan passes close to the cusp of a saddle-node bifurcation. As a complement to the brute force bifurcation diagram in Fig. 3, Fig. 5 shows the results of a one-dimensional continuation scan. Here, we have followed the solutions from the equilibrium point through the Hopf bifurcation and three perioddoubling bifurcations to the period- 8 solution generated at $\alpha \cong 11.7$. Stable solutions are shown as full curves and unstable solutions as broken curves.

A more complete picture of the bifurcation structure of our model is provided by the two-dimensional phase diagram in Fig. 6. Starting around $\alpha=1.3$ for $T=0$, the lowest curve in this diagram is the Hopf bifurcation curve. Below this curve the model displays a stable equilibrium point. The normal operation point around $\alpha=7$ and $T=4.5 \mathrm{~s}$ is just at the edge of this Hopf bifurcation. This seems to explain the experimental finding that about $70 \%$ of the nephrons in a normal rat perform self-sustained oscillations while the remaining show stable equilibrium behavior. We can also imagine that the system is shifted back and forth across the Hopf bifurcation curve by variations in arterial pressure. This

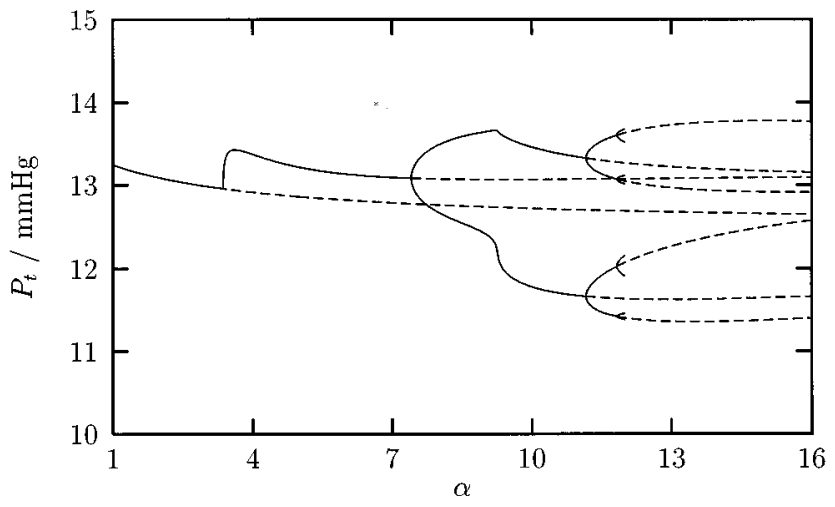

FIG. 5. One-dimensional continuation scan for $T=3 \mathrm{~s}$. This diagram follows the variation of the equilibrium point and of the period-1, $-2,-4$, and -8 solutions through the regions where they are stable (full curves) as well as where they are unstable (dotted curves).

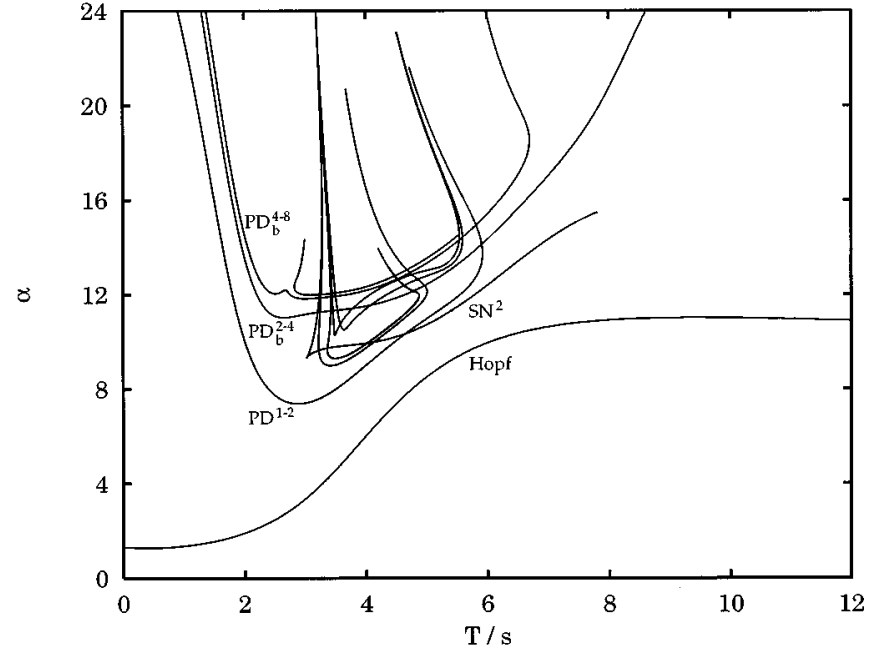

FIG. 6. Two-dimensional phase diagram illustrating the overlying perioddoubling cascades in the nephron model. The normal operation point is close to $\alpha=7$ and $T=4.5 \mathrm{~s}$.

would explain the temporal behavior of the nephrons with periods of self-sustained oscillations interrupted by periods of stable behavior.

In accordance with Fig. 3, a vertical scan in Fig. 6 for $T=3$ s first passes the Hopf bifurcation curve at $\alpha \cong 3.5$. At $\alpha \cong 7.5$ we meet the $P D^{1-2}$ period-doubling curve in which the stable period-2 solution is born. Hereafter the $P D_{b}^{2-4}$ and $P D_{b}^{4-8}$ curves follow in which stable period-4 and period-8 solutions are born. We also note how the scan for $\alpha \cong 9.3$ passes very close to the cusp of the $S N^{2}$ saddle-node bifurcation curve.

The details of the bifurcation diagram are more easily resolved in Fig. 7, which is a magnification of the center part of Fig. 6. This magnification reveals a variant of the characteristic structure of crossing fold and flip bifurcations previously observed, for instance, for a variety of one- and two-

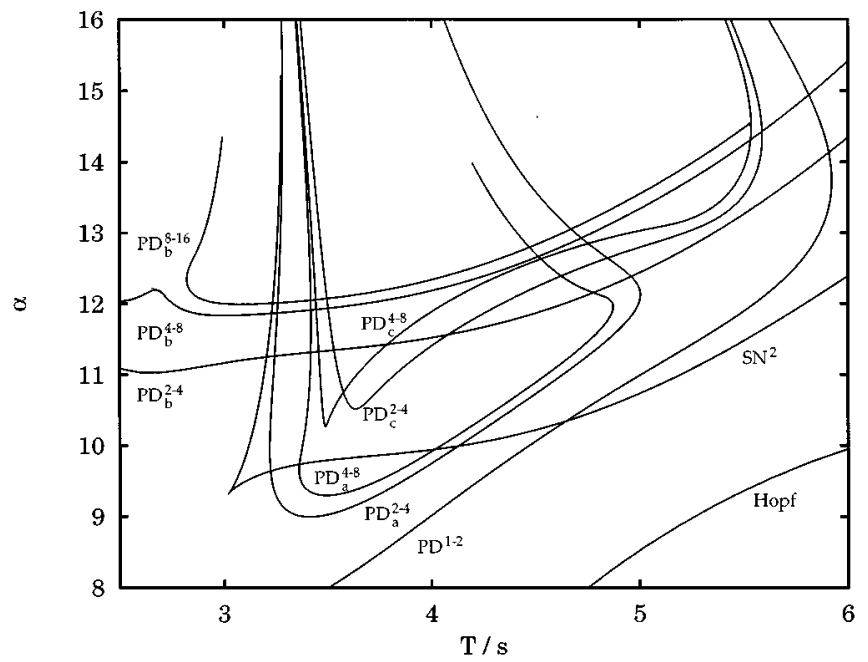

FIG. 7. Magnification of part of the bifurcation diagram in Fig. 6. Note the overlying structure of several different period-doubling cascades. This is a variant of the so-called crossroad structure. 




FIG. 8. One-parameter bifurcation scan from $\alpha=8, T=4$ s to $\alpha=14, T=2.5$ $\mathrm{s}$. Note how the saddle-node bifurcations fold the period-doubling sequences on top of one another.

dimensional maps. ${ }^{27-29}$ This structure consists of perioddoubling cascades folded on top of one another by saddlenode bifurcations.

As illustrated in Fig. 8, a linear scan from $\alpha=8, T=4 \mathrm{~s}$ toward $\alpha=14, T=2.5$ s first takes us across the $P D^{1-2}$ bifurcation curve at which the first period-doubling occurs. We hereafter cross the lower branches of the $P D_{a}^{2-4}$ and $P D_{a}^{4-8}$ curves at which stable period-4 and period- 8 solutions appear. The scan continues through the upper branch of the $P D_{a}^{4-8}$ curve to meet the upper branch of a $S N^{4}$ saddle-node bifurcation curve. Here, the stable period-4 solution destabilizes. The period- 4 saddle can be followed backward to the lower branch of the same $S N^{4}$ curve, where it again stabilizes. The stable period-4 solution passes through another $P D^{4-8}$ flip bifurcation and two reverse flip bifurcations to reestablish a stable period-2 solution immediately before it reaches the upper branch of the $S N^{2}$ curve. Here, the period-2 solution is destabilized in a saddle-node bifurcation. The unstable period-2 solution can be followed backward to the lower branch of the $S N^{2}$ curve, where it regains stability in a new saddle-node bifurcation. The stable period- 2 solution formed in this bifurcation can hereafter be followed up through the $P D_{b}^{2-4}, P D_{b}^{4-8}$, and $P D_{b}^{8-16}$ period-doubling curves into the chaotic regime in the top left corner of the diagram. On the way it passes the tip of a new $S N^{4}$ saddle node and crosses another $P D^{8-16}$ period-doubling curve.

Besides these period-doubling sequences, the nephron model exhibits an additional period-doubling cascade (indicated by index $c$ ). It is observed in Fig. 7 how the perioddoubling curves associated with the $c$ cascade cross the period-doubling curves associated with the other cascades. Hence, there must be additional saddle-node bifurcations folding these bifurcation cascades on top of one another. It is also noted that the $P D_{c}^{4-8}$ curve crosses the $P D_{c}^{2-4}$ curve. This implies the existence of an $S N^{4}$ saddle-node bifurcation curve folding the period-4 solution and ending in a flip codimension-2 bifurcation point near the minimum of the $P D_{c}^{2-4}$ curve. To the right of this point, the $2-4$ period doubling is supercritical. To the left the period doubling is subcritical, and the period-4 solution is born as an unstable solution.

\section{DISCUSSION}

We have analyzed the bifurcation structure of a relatively detailed model of the tubuloglomerular feedback mechanism in kidney pressure and flow control. Almost all experimental work on this problem relates to rats, and the model parameters also pertains specifically to this system. It is supposed, however, that similar phenomena take place in the human kidney, and that they play a major role for the development of high blood pressure. A better understanding of these phenomena may therefore be significant for the treatment of some of the cardiovascular diseases that plague our industrialized societies.

With physiologically based relations between the various variables, and with realistic parameter values, the model reproduces many of the experimental findings. First of all, the model can perform self-sustained oscillations with the correct period and amplitude. Second, by operating close to a Hopf threshold the model explains why some nephrons oscillate and others do not. Finally, the model can produce period doubling and chaos as observed in certain experiments. Unfortunately, one cannot determine the parameter values pertaining to the specific nephron under study, and for this reason it is not possible directly to relate experimental results to the theoretical predictions.

From a physical point of view the observed nonlinear dynamic phenomena derive from the fact that the nephron is a thermodynamically open system that operates far from thermal equilibrium. The heart continues to pump blood through the afferent/efferent arteriolar system and hereby maintains the hydrostatic pressure difference required for filtration to take place. In addition, the epithelial cells in the tubular wall depend on a steady supply of metabolic energy to perform their active secretion of foreign chemicals into and reabsorption of salts from the filtrate. From a control theoretical point of view, the instability may be ascribed to the steepness of the regulatory characteristics. If the tubuloglomerular feedback depended less strongly on the Henle flow, the system would be stable.

The most obvious shortcoming of the model in its present state appears to be the lack of a proper account of the myogenic response of the first section of the arteriolar wall. It is likely that this response interacts with the tubuloglomerular response. If, for instance, a TGF-mediated constriction has caused the lower part of the afferent arteriole to contract, the hydrostatic pressure in the upper part of the vessel will increase. This will cause that part of the vessel to contract, and hence further increase the hemodynamic resistance of the arteriole. It is also likely that the myogenic response plays a major role in the coupling of neighboring nephrons.

\section{ACKNOWLEDGMENTS}

We are grateful to R. Feldberg for his contributions to the initial phases of this analysis. R. Feldberg, C. Knudsen, and J. S. Thomsen developed the computer software applied in the continuation studies. 
${ }^{1}$ L. Glass and M. C. Mackey, From Clocks to Chaos, The Rhythms of Life (Princeton University Press, Princeton, NJ, 1988).

${ }^{2}$ E. Mosekilde and O. G. Mouritsen, Modeling the Dynamics of Biological Systems, Nonlinear Phenomena and Pattern Formation (Springer-Verlag, Berlin, 1995).

${ }^{3}$ W. F. Crowley and J. G. Hofler, The Episodic of Hormones (Wiley, New York, 1987).

${ }^{4}$ G. Leng, Pulsatility in Neuroendocrine Systems (CRC Press, Boca Raton, FL, 1988).

${ }^{5}$ J. E. Levine, Pulsatility in Neuroendocrine Systems, Methods in Neurosciences (Academic, New York, 1994), Vol. 20.

${ }^{6}$ A. Goldbeter, Cell to Cell Signalling: From Experiments to Theoretical Models (Academic, London, 1989).

${ }^{7}$ L. C. Moore, "Tubuloglomerular feedback and SNGFR autoregulation in the rat," Am. J. Physiol. 247, F267-F276 (1984).

${ }^{8}$ P. P. Leyssac and L. Baumbach, "An oscillating intratubular pressure response to alterations in Henle loop flow in the rat kidney," Acta Physiol. Scand. 117, 415-419 (1983).

${ }^{9}$ N.-H. Holstein-Rathlou and P. P. Leyssac, "TGF-mediated oscillations in the proximal intratubular pressure: Difference between spontaneous hypertensive rats and Wistar-Kyoto rats," Acta. Physiol. Scand. 126, 333339 (1986)

${ }^{10}$ P. P. Leyssac and N.-H. Holstein-Rathlou, "Effects of various transport inhibitors on oscillating TGF pressure responses in the rate," Pfluegers Archiv. 407, 285-291 (1986).

${ }^{11}$ K.-P. Yip, N.-H. Holstein-Rathlou, and D. J. Marsh, "Chaos in blood flow control in genetic and renovascular hypertensive rats," Am. J. Physiol. 261, F400-F408 (1991).

${ }^{12}$ N.-H. Holstein-Rathlou and P. P. Leyssac, "Oscillations in the proximal intratubular pressure: A mathematical model," Am. J. Physiol. 252, F560-F572 (1987).

${ }^{13}$ K. S. Jensen, E. Mosekilde, and N.-H. Holstein-Rathlou, "Self-sustained oscillations and chaotic behavior in kidney pressure regulation," Mondes Develop. 54/55, 91-109 (1986).

${ }^{14}$ K. S. Jensen, N.-H. Holstein-Rathlou, P. P. Leyssac, E. Mosekilde, and D. R. Rasmussen, "Chaos in a system of interacting nephrons," in Chaos in Biological Systems, edited by H. Degn, A. V. Holden, and L. F. Olsen (Plenum, New York, 1987), pp. 23-32

${ }^{15}$ N.-H. Holstein-Rathlou, "Synchronization of proximal intratubular pressure oscillations: Evidence for interaction between nephrons," Pfluegers Archiv. 408, 438-443 (1987).

${ }^{16}$ N.-H. Holstein-Rathlou and D. J. Marsh, "Oscillations of tubular pressure, flow, and distal chloride concentration in rats," Am. J. Physiol. 256, F1007-F1014 (1989).

${ }^{17}$ N.-H. Holstein-Rathlou and D. J. Marsh, " A dynamic model of the tubuloglomerular feedback mechanism," Am. J. Physiol. 258, F1448-F1459 (1990).
${ }^{18}$ N.-H. Holstein-Rathlou, A. J. Wagner, and D. J. Marsh, "Tubuloglomerular feedback dynamics and renal blood flow autoregulation in rats," Am. J. Physiol. 260, F53-F68 (1991).

${ }^{19}$ N.-H. Holstein-Rathlou and D. J. Marsh, "A dynamic model of renal blood flow autoregulation,' Bull. Math. Biol. 56, 411-429 (1994).

${ }^{20}$ R. Feldberg, M. Colding-Jørgensen, and N.-H. Holstein-Rathlou, "An analysis of the interaction between tubuloglomerular feedback and the myogenic response in renal blood flow autoregulation,' Am. J. Physiol. 269, F581-F593 (1995).

${ }^{21}$ C. Casellas and L. C. Moore, "Autoregulation and tubuloglomerular feedback in juxtamedullary glomerular arterioles," Am. J. Physiol. 258, F666-F669 (1990).

${ }^{22}$ D. A. Haberle and J. M. Davis, "Interrelationship between proximal tubular hydrodynamics and tubuloglomerular feedback in the rat kidney," Kidney Int. 22, Suppl. 12, S193-S197 (1982).

${ }^{23}$ J. Briggs, "A simple steady-state model for feedback control of glomerular filtration rate," Kidney Int. 22, Suppl. 12, S143-S150 (1982).

${ }^{24}$ R. Feldberg, M. Colding-Jørgensen, and N.-H. Holstein-Rathlou, "A model of the myogenic response in small arteries and arterioles," in Proceedings of the 1st Copenhagen Symposium on Computer Simulation in Biology, Ecology and Medicine, edited by E. Mosekilde (The Society for Computer Simulation, San Diego, 1993), pp. 110-114.

${ }^{25}$ L. O. Chua, M. Komuro, and T. Matsumoto, "The double scroll family, parts I and II," IEEE Trans. Circ. Syst. CAS-33, 1072-1118 (1986).

${ }^{26}$ L. Glass and R. Perez, "Fine structure of phase locking," Phys. Rev. Lett. 48, 1772-1775 (1982).

${ }^{27}$ M. Schell, S. Fraser, and R. Kapral, "Subharmonic bifurcation in the sine map: An infinite hierarchy of cusp bistabilities," Phys. Rev. A 28, 373378 (1983).

${ }^{28}$ J. P. Carcasses, C. Mira, M. Bosch, C. Simó, and J. C. Tatjer, "Crossroad area-Spring area transition (I) parameter plane representation," Int. J. Bifurc. Chaos 1, 183-196 (1991)

${ }^{29}$ A. P. Kuznetsov, S. P. Kuznetsov, and I. R. Sataev, "From bimodal onedimensional maps to Hénon-like two-dimensional maps: Does quantitative universality survive?," Phys. Lett. A 184, 413-421 (1994).

${ }^{30}$ R. S. MacKay and C. Tresser, "Some flesh on the skeleton: The bifurcation structure of bimodal maps," Physica D 27, 412-422 (1987).

${ }^{31}$ M. Rosenbaum and D. Race, "Frequency-response characteristics of vascular resistance vessels," Am. J. Physiol. 215, 1397-1402 (1968).

${ }^{32}$ Y.-C. B. Fung, Biomechanics. Mechanical Properties of Living Tissues (Springer, New York, 1981).

${ }^{33}$ R. J. Paul, "Smooth muscle: Mechano-chemical energy conversion relations between metabolism and contractibility," in Physiology of the Gastrointestinal Tract, edited by L. R. Johnson (Raven, New York, 1981), pp. 269-288. 